\title{
Efecto de poda y raleo en el área foliar de Eucalyptus nitens
}

\author{
Effect of pruning and thinning intensity on Eucalyptus nitens leaf area
}

\author{
Fernando Muñoz ${ }^{\mathrm{a}}$, Miguel Espinosa ${ }^{\mathrm{a}}$, Jorge Cancino ${ }^{\mathrm{a}}$, \\ Rafael Rubilar a, Miguel Herrera ${ }^{b}$

\begin{abstract}
*Autor de correspondencia: aUniversidad de Concepción, Facultad de Ciencias Forestales, Victoria 631, casilla 160-C, Concepción, Chile, tel.: 41-2204679, fax: 41-2246004,fmunoz@udec.cl bUniversidad de Córdoba, Escuela Técnica Superior de Ingenieros Agrónomos y de Montes, España.
\end{abstract}

\begin{abstract}
SUMMARY
Eucalyptus nitens is an important commercial fast growing species planted mainly in Chile and Australia (Tasmania). In Chile there are not previously published studies quantifying leaf area in adult forest plantations of $E$. nitens. This study presents the effect of pruning and thinning intensities, applied at age 6 in a E. nitens stand, and on the amount of leaf area sustained at 15 years old. A factorial experiment considering pruning $\left(0 \mathrm{~m}, 3.5 \mathrm{~m}\right.$ and $7 \mathrm{~m}$ height) and stocking $\left(1,100,800\right.$ and 400 trees ha $\left.^{-1}\right)$ was established in 1989 near Los Álamos town in central Chile. Using the average tree method, 27 trees were sampled destructively for biomass and leaf area assessments. Projected leaf area, leaf area index, and specific leaf area were estimated for each sampled tree. The results indicate that projected leaf area and leaf area index reached a maximum of $64.7 \mathrm{~m}^{2}$ tree $^{-1}$ at 400 trees ha ${ }^{-1}$ stocking and 3.48 $\mathrm{m}^{2} \mathrm{~m}^{-2}$ at 800 trees ha-1 stocking respectively. Specific leaf area was higher $\left(43.2 \mathrm{~cm}^{2} \mathrm{~g}^{-1}\right)$ at higher stocking $(1,100$ trees ha-1). For all treatments projected leaf area and leaf area index were higher in the central section of the crown. Allometric relationships were obtained using diameter at the base of the live crown and stump diameter. The information presented in this study allows giving a more functional explanation of the horizontal and vertical behavior of the leaf area, at different stocking.
\end{abstract}

Key words: Eucalyptus nitens, leaf area, pruning, thinning.

RESUMEN

Eucalyptus nitens es una especie de rápido crecimiento plantada principalmente en Chile y Australia (Tasmania). En Chile, no hay estudios publicados de área foliar en bosques adultos de E. nitens. Se investigó el efecto de la poda y el raleo en el área foliar de E. nitens. Los datos utilizados provienen de una plantación de 15 años de edad, luego de nueve años de establecido el ensayo. El estudio fue realizado en una plantación efectuada el año 1989, cercana a la localidad de Los Álamos, zona central de Chile, según un arreglo factorial de dos factores: intensidad de raleo y severidad de poda. Utilizando el método del árbol medio, se seleccionaron 27 árboles que posteriormente fueron volteados. Con las mediciones realizadas se obtuvo el área foliar proyectada, índice de área foliar y área foliar específica. El análisis de varianza evidenció un efecto significativo del raleo sobre el área foliar. El área foliar proyectada e índice de área foliar es máxima en los tratamientos de densidad 400 árboles ha ${ }^{-1}\left(64,7 \mathrm{~m}^{2}\right.$ árbol $\left.{ }^{-1}\right)$ y en tratamientos de densidad 800 árboles $\mathrm{ha}^{-1}\left(3,48 \mathrm{~m}^{2} \mathrm{~m}^{-2}\right)$, respectivamente. El área foliar específica es mayor en tratamientos de densidad 1.100 árboles $\mathrm{ha}^{-1}\left(43,2 \mathrm{~cm}^{2} \mathrm{~g}^{-1}\right)$. El área foliar proyectada e índice de área foliar se concentran en la sección central de la copa. Los modelos alométricos determinados utilizan como variable predictora el diámetro en la base de la copa viva y el diámetro de tocón. La información presentada en el estudio permite dar una explicación más funcional del comportamiento horizontal y vertical del área foliar, a diferentes densidades residuales.

Palabras clave: Eucalyptus nitens, área foliar, poda, raleo.

\section{INTRODUCCIÓN}

La cantidad de luz interceptada por el follaje es importante para el crecimiento de los árboles (Beadle et al. 1995) y, por lo tanto, para la productividad y dinámica de los rodales (Oliver y Larson 1996). El índice de área foliar (IAF) relaciona la extensión del follaje por unidad de superficie (Perry 1994, Cherry et al. 1998, Smethurst et al. 2003), siendo considerado un buen indicador de la capacidad de la copa para absorber la radiación fotosintéticamente activa (Perry 1994).
Los métodos directos de estimación de área foliar, como muestreo destructivo de árboles y ramas, son lentos y costosos, pero de mayor precisión (Cherry et al. 2002) que los métodos indirectos, como relaciones alométricas entre área foliar y características del árbol (Norman y Campbell 1989), uso de radiómetros del tipo Li-cor Lai2000 (Cherry et al. 1998, Battaglia et al. 1998), fotografía hemisférica (Keane et al. 2005) y guías visuales para su uso comparativo al interior de la plantación (Cherry et al. 2002). 
El área foliar está fuertemente relacionada con el nivel de interceptación de luz, transpiración y fotosíntesis neta en la copa, y varía de acuerdo a las condiciones ambientales en las cuales se desarrolla un rodal (Battaglia et al. 1998, Amponsah et al. 2005), a la edad (Jerez et al. 2005), a la época del año (Simioni et al. 2004) y a las características de éste (Rodríguez 2002). Por lo tanto, la estimación del área foliar puede ser usada como una herramienta de manejo de gran valor para monitorear y predecir el crecimiento de la plantación (Cherry et al. 2002), aspecto de importancia en el manejo de plantaciones de rápido crecimiento.

La mayoría de los modelos de crecimiento de procesos consideran como indicador de la interceptación de luz el área foliar horizontal de la vegetación. Ésta es una aproximación adecuada cuando hay cierre total de copas, pero no lo es cuando hay discontinuidad de copas, como resultado de intervenciones de raleos (Medhurst y Beadle 2001).

Eucalyptus nitens (Deane et Maiden) Maiden es una especie de rápido crecimiento, plantada principalmente en Chile y Australia (Tasmania), con una superficie que representa el 88\% (300.000 ha) del total mundial (340.000 ha) (INFOR 2004). En estos países, E. nitens se utiliza mayoritariamente para pulpa (Smethurst et al. 2003, INFOR 2004). Sin embargo, en Australia, desde hace algunos años, se han incorporado podas y raleos en el manejo de las plantaciones que permitan generar madera de mayor calidad (Pinkard et al. 1995). En Chile, el manejo de E. nitens no es una práctica frecuente (INFOR 2004, Muñoz et al. 2005), aunque se está produciendo un cambio en este aspecto tendiente a obtener productos de mayor calidad como madera aserrada y chapas, lo cual requiere de podas y raleos.

Los objetivos de este estudio fueron a) determinar el efecto de la poda y el raleo en el área foliar y su distribución en la copa para plantaciones de E. nitens y b) establecer relaciones alométricas entre área foliar y variables asociadas al crecimiento.

\section{MÉTODOS}

El ensayo se estableció en la localidad de Los Álamos (373' S, 7327' O), provincia de Arauco, Región del Bío Bío, zona central de Chile, en un rodal de E. nitens de 4,2 ha de superficie. El sitio está localizado a $180 \mathrm{~m}$ de elevación y presenta topografía de lomaje suave. El suelo es originado a partir de sedimentos marinos, clasificados en el orden Alfisol, suborden Xeralfs. Son suelos de textura franco arcillosa, ligeramente plásticos y adhesivos en húmedo, duros y compactos en seco (Carrasco y Millán 1990). El análisis químico de suelo realizado determinó $\mathrm{pH} 5,04,3,25 \%$ de carbono total, $0,2 \%$ de nitrógeno total, relación $\mathrm{C} / \mathrm{N}$ de 16 y 2,0 $\mathrm{mg} \mathrm{kg}^{-1}$ de fósforo disponible.

La temperatura varía entre una media máxima en el mes de enero de $23,2^{\circ} \mathrm{C}$ y una media mínima en julio de $6,0{ }^{\circ} \mathrm{C}$. La precipitación promedio anual es de $1.437 \mathrm{~mm}$ con un período seco de cinco meses, y la radiación solar promedio anual es de 327 Ly día $^{-1}$ (Santibáñez y Uribe 1993) (figura 1).



Figura 1. Valores medios mensuales de precipitación $(\mathrm{mm})$, temperatura media $\left({ }^{\circ} \mathrm{C}\right)$ y radiación solar $\left(\mathrm{Ly} \mathrm{día}^{-1}\right)$, para el sector Los Álamos según Santibáñez y Uribe (1993).

Total monthly precipitation $(\mathrm{mm})$, mean monthly temperature $\left({ }^{\circ} \mathrm{C}\right)$, total daily solar radiation (Ly/day) for Los Alamos site by Santibáñez y Uribe (1999).

La plantación se realizó en el invierno de 1989 con un espaciamiento de 2,0 x 3,5 m, sobre líneas de subsolado, con plantas producidas de semillas de procedencia Toorongo (Victoria, Australia). Previo a la plantación se efectuó control de malezas. El cultivo anterior fue una plantación de pino radiata (Pinus radiata D. Don), cuyos residuos de cosecha fueron quemados.

Diseño experimental y mediciones. El diseño experimental correspondió a un arreglo factorial de dos factores (altura de poda e intensidad de raleo) en tres niveles cada uno. Los tratamientos fueron establecidos en octubre de 1995 y resultaron de la combinación de tres densidades nominales de rodal (1.100 [sin raleo], 800 y 400 árboles ha $^{-1}$ ) y tres alturas de poda ( 0 [sin poda], 3,5 y 7,0 m). Por cada tratamiento (nueve en total) se realizaron tres repeticiones, con un total de 27 parcelas, a las que se les asignaron los tratamientos en forma aleatoria. Se establecieron parcelas cuadradas de $324 \mathrm{~m}^{2}(18 \times 18 \mathrm{~m})$ rodeadas por una faja perimetral de aislamiento de $5 \mathrm{~m}$ de ancho.

En octubre de 2004 se seleccionó aleatoriamente en cada parcela un árbol que correspondiera a la clase de diámetro medio (Pardé 1980). En total se seleccionaron 27 árboles (tres árboles por cada tratamiento). Previo al volteo de los árboles, se midió el DAP a cada uno. Una 
vez derribados, se midieron el diámetro de tocón, el diámetro del fuste en la base de copa viva, la longitud de copa viva y la altura total. La copa fue dividida en tercios de igual longitud. Para cada tercio se determinó el peso verde total de hojas. Se obtuvo una muestra de hojas de cada tercio y se determinó el peso verde. En laboratorio las muestras fueron secadas al horno a $75{ }^{\circ} \mathrm{C}$ por 48 horas. Una submuestra de hojas verdes de cada tercio fue usada para determinar la superficie foliar utilizando el medidor Li-Cor 3100 (Li-Cor Inc., Lincoln, Nebraska, USA); las submuestras fueron secadas y se determinó su peso seco. Se calculó el área foliar específica (AFE) de cada submuestra, como la razón área foliar verde/biomasa foliar seca. La biomasa foliar de cada tercio de la copa fue determinada multiplicando el peso verde por la razón peso seco de hojas/peso verde de hojas. El índice de área foliar (IAF) del árbol completo correspondió a la sumatoria de las áreas foliares de sus respectivos tercios, proyectada sobre el área media de suelo que ocupan los árboles en la parcela. El área foliar proyectada (AFP) de cada tercio de la copa fue calculada multiplicando su biomasa foliar por el AFE.

Análisis de datos. Todas las variables fueron sometidas a pruebas para determinar su normalidad y homogeneidad de varianzas. El efecto de los tratamientos sobre la magnitud y distribución del IAF, AFP y AFE se determinó mediante análisis de varianza $(P<0,05)$. La separación de medias, cuando correspondía, se realizó utilizando la prueba de Tukey (Steel y Torrie 1988). Mediante análisis de regresión lineal se ajustaron las variables IAF, AFP y AFE como variables dependientes y las variables predictoras DAP, diámetro del tocón, diámetro en la base de la copa viva, largo de la copa viva, altura total, además de sus transformaciones cuadráticas y logarítmicas. En el proceso de datos se utilizó el programa estadístico StatSoft (2001).

\section{RESULTADOS}

Como era de esperar, el tratamiento sin poda y sin raleo (T0) presentó la menor área foliar proyectada (AFP) $\left(14,6 \mathrm{~m}^{2}\right.$ árbol $\left.^{-1}\right)$ y el tratamiento con poda a 7 $\mathrm{m}$ y raleo a 400 árboles ha ${ }^{-1}$ (T8, tratamiento más intenso) obtuvo el valor más alto de AFP $\left(77,0 \mathrm{~m}^{2}\right.$ árbol $\left.^{-1}\right)$ (cuadro 1). En todos los tratamientos, la mayor AFP se concentró en la sección central de la copa. El AFP fue afectado significativamente por el raleo, siendo mayor en tratamientos de densidad 400 árboles ha ${ }^{-1}$ (cuadro 2). La poda no tuvo efecto significativo sobre el AFP total o sobre el AFP de cada sección de copa. La interacción entre raleo y poda tuvo efecto significativo en el AFP total $(P=0,0226)$ y el AFP de la base de la copa $(P=0,0279)$. Para todos los niveles de raleo y poda estudiados, el mayor AFP se concentró en la sección central de la copa (cuadro 2).
Cuadro 1. Área foliar proyectada (AFP) según sección de copa y tratamiento.

Projected leaf area (AFP) by crown section and treatment.

\begin{tabular}{|c|c|c|c|c|c|c|}
\hline \multicolumn{3}{|c|}{ Tratamientos } & \multicolumn{4}{|c|}{$\begin{array}{c}\text { Área foliar } \\
\text { proyectada }\left(\mathrm{m}^{2} \text { árbol }{ }^{-1}\right)\end{array}$} \\
\hline $\begin{array}{c}\text { Raleo } \\
\text { (árboles ha-1) }\end{array}$ & $\begin{array}{l}\text { Poda } \\
\text { (m) }\end{array}$ & Código & Ápice & Centro & Base & Total \\
\hline 400 & 0 & T6 & 10,2 & $39,0 \mathrm{a}$ & $24,0 \mathrm{ab}$ & $73,2 \mathrm{a}$ \\
\hline 400 & 3,5 & $\mathrm{~T} 7$ & 11,0 & 22,1 abcd & $12,0 \mathrm{bc}$ & 43,9abc \\
\hline 400 & 7 & $\mathrm{~T} 8$ & 9,8 & $33,4 a b$ & $32,6 a$ & $77,0 \mathrm{a}$ \\
\hline 800 & 0 & $\mathrm{~T} 3$ & 7,6 & $19,2 \mathrm{bcd}$ & $9,3 \mathrm{bc}$ & $36,0 \mathrm{bc}$ \\
\hline 800 & 3,5 & $\mathrm{~T} 4$ & 14,0 & $23,5 \mathrm{abcd}$ & $12,7 \mathrm{bc}$ & $50,1 \mathrm{ab}$ \\
\hline 800 & 7 & T5 & 10,1 & $25,5 \mathrm{abc}$ & $8,2 \mathrm{bc}$ & $43,8 \mathrm{abc}$ \\
\hline 1.100 & 0 & T0 & 3,7 & $7,1 \mathrm{~d}$ & $3,9 \mathrm{c}$ & $14,6 \mathrm{c}$ \\
\hline 1.100 & 3,5 & $\mathrm{~T} 1$ & 5,6 & $10,5 \mathrm{~cd}$ & $6,2 \mathrm{c}$ & $22,3 b c$ \\
\hline 1.100 & 7 & $\mathrm{~T} 2$ & 2,7 & $8,6 \mathrm{~cd}$ & $8,3 \mathrm{bc}$ & $19,5 b c$ \\
\hline
\end{tabular}

En cada columna, letras diferentes indican diferencias significativas (Tukey, $P<0,05$ ).

Cuadro 2. Área foliar proyectada (AFP) según sección de copa y nivel de intervención de raleo y poda.

Projected leaf area (AFP) by crown section at different stocking and pruning intensities.

\begin{tabular}{|c|c|c|c|c|c|}
\hline \multirow{2}{*}{\multicolumn{2}{|c|}{ Factores }} & \multicolumn{4}{|c|}{ Área foliar proyectada $\left(\mathrm{m}^{2}\right.$ árboles $\left.{ }^{-1}\right)$} \\
\hline & & Ápice & Centro & Base & Total \\
\hline \multirow[t]{3}{*}{ Raleo } & 400 & $10,3 \mathrm{a}$ & $31,5 \mathrm{a}$ & $22,9 \mathrm{a}$ & $64,7 \mathrm{a}$ \\
\hline & 800 & $10,6 \mathrm{a}$ & $22,7 \mathrm{~b}$ & $10,0 \mathrm{~b}$ & $43,3 b$ \\
\hline & 1.100 & $4,0 \mathrm{~b}$ & $8,7 \mathrm{c}$ & $6,1 b$ & $18,8 \mathrm{c}$ \\
\hline \multirow[t]{3}{*}{ Poda } & 0 & $7,1 \mathrm{a}$ & $21,8 \mathrm{a}$ & $12,4 \mathrm{a}$ & $41,3 \mathrm{a}$ \\
\hline & 3,5 & $9,8 \mathrm{a}$ & $18,7 \mathrm{a}$ & $10,3 \mathrm{a}$ & $38,8 \mathrm{a}$ \\
\hline & 7 & $7,9 \mathrm{a}$ & $22,6 \mathrm{a}$ & $16,3 \mathrm{a}$ & $46,8 \mathrm{a}$ \\
\hline \multicolumn{2}{|c|}{ Raleo $(P)$} & $0,0055^{* *}$ & $<0,0001 * * *$ & $<0,0001 * * *$ & $<0,0001 * * *$ \\
\hline \multicolumn{2}{|c|}{ Poda $(P)$} & $0,4192 \mathrm{~ns}$ & $0,4094 \mathrm{~ns}$ & $0,1378 \mathrm{~ns}$ & $0,3590 \mathrm{~ns}$ \\
\hline \multicolumn{2}{|c|}{$\begin{array}{r}\text { Raleo } \mathrm{x} \\
\text { poda }(P)\end{array}$} & $0,6403 \mathrm{~ns}$ & $0,0513 \mathrm{~ns}$ & $0,0279 *$ & $0,0226 *$ \\
\hline
\end{tabular}

$P$ : probabilidades límites en ANOVA con dos factores. *: $P<0,05$; **: $P<0,01$; ***: $P<0,001$; ns: $P>0,05$.

Letras diferentes en cada columna indican diferencias significativas (Tukey, $P<0,05$ ).

Factores: factor raleo en árboles $\mathrm{ha}^{-1} \mathrm{y}$ factor poda en metros.

El índice de área foliar (IAF) promedio por árbol del tratamiento poda a $3,5 \mathrm{~m}$ y raleo a 800 árboles ha ${ }^{-1}$ (T4) fue de $3,84 \mathrm{~m}^{2} \mathrm{~m}^{-2}$, significativamente mayor al tratamiento T0 $\left(1,63 \mathrm{~m}^{2} \mathrm{~m}^{-2}\right)$, siendo los únicos tratamientos que presentaron diferencias significativas (cuadro 3). En todos los tratamientos, la mayor proporción del IAF por árbol se concentró en el tercio central de la copa. El área foliar específica (AFE) (cuadro 3) no presentó diferen- 
cias significativas entre tratamientos. El mayor valor se alcanzó en el tratamiento T0 $\left(46,0 \mathrm{~cm}^{2} \mathrm{~g}^{-1}\right)$ y el menor en el tratamiento poda a $7 \mathrm{~m}$ y raleo a 800 árboles ha ${ }^{-1}$ (T5) con $36,6 \mathrm{~cm}^{2} \mathrm{~g}^{-1}$. En los tratamientos de densidad 400 árboles ha ${ }^{-1}$, el AFE fue mayor en la base de la copa. En los restantes tratamientos no se observó una tendencia clara (cuadro 3).

El factor raleo afectó el IAF del ápice y centro de la copa $(P=0,005$ y 0,0012 , respectivamente) y el AFE del ápice $(P=0,0353)$ (cuadro 4). A diferencia del raleo, la poda no afectó el IAF, como tampoco el AFE, independientemente del nivel de raleo aplicado. No se detectó evidencia de interacción entre poda y raleo.
Las variables predictoras que mejor explicaron la variable dependiente IAF fueron el diámetro de tocón (DT) y el diámetro en la base de la copa viva (DBCV). Para el AFE, el DT; y para el AFP, el DBCV, transformado en su expresión logarítmica. El modelo alométrico para estimar AFP presentó mejor correlación que los modelos para estimar IAF y AFE. El coeficiente de determinación $\left(\mathrm{R}^{2}\right)$ fluctuó entre 0,21 para el modelo que estima el índice área foliar y 0,82 para el modelo que estima el área foliar proyectada (cuadro 5). Los modelos alométricos obtenidos presentaron residuos que variaron de manera aleatoria alrededor de la línea base.

Cuadro 3. Efecto de los tratamientos en el índice de área foliar (IAF) y área foliar específica (AFE).

Effect of treatments in the leaf area index (IAF) and specific leaf area (AFE).

\begin{tabular}{|c|c|c|c|c|c|c|c|c|c|c|}
\hline \multicolumn{3}{|c|}{ Tratamientos } & \multicolumn{4}{|c|}{ Índice de área foliar $\left(\mathrm{m}^{2} \mathrm{~m}^{-2}\right)$} & \multicolumn{4}{|c|}{ Área foliar específica $\left(\mathrm{m}^{2} \mathrm{~g}^{-1}\right)$} \\
\hline $\begin{array}{c}\text { Raleo } \\
\left(\text { árboles } \mathrm{ha}^{-1}\right)\end{array}$ & $\begin{array}{l}\text { Poda } \\
(\mathrm{m})\end{array}$ & Código & Ápice & Centro & Base & Total & Ápice & Centro & Base & Total \\
\hline 400 & 0 & T6 & $0,44 \mathrm{ab}$ & $1,68 \mathrm{ab}$ & 1,04 & $3,16 \mathrm{ab}$ & 37,3 & 39,4 & 40,0 & 38,9 \\
\hline 400 & 3,5 & $\mathrm{~T} 7$ & $0,42 \mathrm{ab}$ & $0,93 \mathrm{ab}$ & 0,50 & $1,84 \mathrm{ab}$ & 40,4 & 40,7 & 44,1 & 41,6 \\
\hline 400 & 7 & $\mathrm{~T} 8$ & $0,44 \mathrm{ab}$ & $1,34 \mathrm{ab}$ & 1,31 & $3,09 \mathrm{ab}$ & 36,7 & 38,1 & 38,9 & 38,0 \\
\hline 800 & 0 & $\mathrm{~T} 3$ & $0,64 \mathrm{ab}$ & $1,61 \mathrm{ab}$ & 0,78 & $3,03 \mathrm{ab}$ & 37,6 & 37,6 & 37,2 & 37,4 \\
\hline 800 & 3,5 & $\mathrm{~T} 4$ & $1,05 \mathrm{~b}$ & $1,82 \mathrm{ab}$ & 0,97 & $3,84 b$ & 40,7 & 40,9 & 43,2 & 41,6 \\
\hline 800 & 7 & $\mathrm{~T} 5$ & $0,82 \mathrm{ab}$ & $2,08 b$ & 0,67 & $3,57 \mathrm{ab}$ & 38,4 & 35,7 & 36,5 & 36,6 \\
\hline 1.100 & 0 & T0 & $0,41 \mathrm{ab}$ & $0,79 \mathrm{a}$ & 0,44 & $1,63 \mathrm{a}$ & 46,9 & 45,4 & 45,8 & 46,0 \\
\hline 1.100 & 3,5 & $\mathrm{~T} 1$ & $0,60 \mathrm{ab}$ & $1,15 \mathrm{ab}$ & 0,68 & $2,44 a b$ & 42,2 & 41,9 & 45,0 & 43,1 \\
\hline 1.100 & 7 & $\mathrm{~T} 2$ & $0,30 \mathrm{a}$ & $0,97 \mathrm{ab}$ & 0,94 & $2,21 \mathrm{ab}$ & 40,4 & 41,5 & 39,6 & 40,4 \\
\hline
\end{tabular}

Letras diferentes en cada columna indican diferencias significativas (Tukey, $P<0,05$ ).

Cuadro 4. Índice de área foliar (IAF) y área foliar específica (AFE) según sección de copa y nivel de intervención de raleo y poda. Leaf area index (IAF) and specific leaf area (AFE) by crown section at different stocking and pruning intensities.

\begin{tabular}{|c|c|c|c|c|c|c|c|c|c|}
\hline \multirow{2}{*}{\multicolumn{2}{|c|}{ Factores }} & \multicolumn{4}{|c|}{ Índice de área foliar $\left(\mathrm{m}^{2} \mathrm{~m}^{-2}\right)$} & \multicolumn{4}{|c|}{ Área foliar específica $\left(\mathrm{m}^{2} \mathrm{~g}^{-1}\right)$} \\
\hline & & Ápice & Centro & Base & Total & Ápice & Centro & Base & Total \\
\hline \multirow[t]{3}{*}{ Raleo } & 400 & $0,43 \mathrm{a}$ & $1,32 \mathrm{a}$ & 0,95 & $2,70 \mathrm{ab}$ & $38,1 \mathrm{a}$ & 39,4 & 41,0 & 39,5 \\
\hline & 800 & $0,84 b$ & $1,84 b$ & 0,81 & $3,48 b$ & $38,9 \mathrm{ab}$ & 38,0 & 39,0 & 38,5 \\
\hline & 1.100 & $0,44 \mathrm{a}$ & $0,97 \mathrm{a}$ & 0,69 & $2,10 \mathrm{a}$ & $43,2 b$ & 42,9 & 43,5 & 43,2 \\
\hline \multirow[t]{6}{*}{ Poda } & 0 & $0,50 \mathrm{a}$ & $1,36 \mathrm{a}$ & 0,75 & $2,61 \mathrm{a}$ & $40,6 \mathrm{a}$ & 40,8 & 41,0 & 40,8 \\
\hline & 3,5 & $0,69 \mathrm{a}$ & $1,30 \mathrm{a}$ & 0,72 & $2,71 \mathrm{a}$ & $41,1 \mathrm{a}$ & 41,2 & 44,1 & 42,1 \\
\hline & 7,0 & $0,52 \mathrm{a}$ & $1,46 \mathrm{a}$ & 0,97 & $2,96 \mathrm{a}$ & $38,5 \mathrm{a}$ & 38,4 & 38,3 & 38,3 \\
\hline & Raleo & $0,0050 * *$ & $0,0012 * *$ & $0,4430 \mathrm{~ns}$ & $0,0046^{* *}$ & 0,0353 & $0,2153 \mathrm{~ns}$ & $0,3455 \mathrm{~ns}$ & $0,1844 \mathrm{~ns}$ \\
\hline & Poda & $0,2520 \mathrm{~ns}$ & $0,7080 \mathrm{~ns}$ & $0,3977 \mathrm{~ns}$ & $0,6220 \mathrm{~ns}$ & $0,3704 \mathrm{~ns}$ & $0,5747 \mathrm{~ns}$ & $0,1854 \mathrm{~ns}$ & $0,3387 \mathrm{~ns}$ \\
\hline & Raleo x Poda & $0,5880 \mathrm{~ns}$ & $0,1599 \mathrm{~ns}$ & $0,1972 \mathrm{~ns}$ & $0,1332 \mathrm{~ns}$ & $0,3860 \mathrm{~ns}$ & $0,8782 \mathrm{~ns}$ & $0,8914 \mathrm{~ns}$ & $0,8033 \mathrm{~ns}$ \\
\hline
\end{tabular}

$P$ : probabilidades límites en ANOVA con dos factores. *: $P<0,05$; **: $P<0,01$; ***: $P<0,001$; ns: $P>0,05$. Letras diferentes en cada columna indican diferencias significativas (Tukey, $P<0,05$ ). Factores: factor raleo en árboles ha ${ }^{-1} \mathrm{y}$ factor poda en metros. 
Cuadro 5. Funciones alométricas por sección y total de copa. Allometric relationships by crown section and whole crown.

\begin{tabular}{lcc}
\hline Funciones alométricas & $\mathrm{R}^{2}$ & $\mathrm{EEE}$ \\
\hline \multicolumn{1}{c}{ Área foliar proyectada } \\
$\ln \left(\mathrm{AFP}_{\mathrm{A}}\right)=-2,3382+1,6574 \ln (\mathrm{DBCV})$ & $0,50 * * *$ & 0,46 \\
$\ln \left(\mathrm{AFP}_{\mathrm{C}}\right)=-2,8150+2,2030 \ln (\mathrm{DBCV})$ & $0,80 * * *$ & 0,30 \\
$\ln \left(\mathrm{AFP}_{\mathrm{B}}\right)=-3,3414+2,1743 \ln (\mathrm{DBCV})$ & $0,53 * * *$ & 0,57 \\
$\ln \left(\mathrm{AFP}_{\mathrm{T}}\right)=-1,8258+2,0963 \ln (\mathrm{DBCV})$ & $0,82 * * *$ & 0,27 \\
\hline
\end{tabular}

Índice de área foliar

$\mathrm{IAF}_{\mathrm{A}}=1,1072-0,0406 \mathrm{DT}+0,0625 \mathrm{DBCV} \quad 0,21 \mathrm{~ns} \quad 0,29$ $\mathrm{IAF}_{\mathrm{C}}=1,1643^{\dagger}-0,0437 \mathrm{DT}^{\dagger}+0,1252 \mathrm{DBCV} 0,32 * * 0,47$ $\mathrm{IAF}_{\mathrm{B}}=0,3298^{\dagger}-0,0191 \mathrm{DT}^{\dagger}+0,0836 \mathrm{DBCV} \quad 0,27 * 0,39$ $\mathrm{IAF}_{\mathrm{T}}=2,6013-0,1034 \mathrm{DT}+0,2713 \mathrm{DBCV} \quad 0,46 * * * 0,74$

Área foliar específica

$\mathrm{AFE}_{\mathrm{A}}=226,8712-10,5169 \mathrm{DT}+0,1453 \mathrm{DT}^{2} \quad 0,73 * * * 2,45$ $\mathrm{AFE}_{\mathrm{C}}=265,5349-12,8613 \mathrm{DT}+0,1803 \mathrm{DT}^{2} 0,61 * * * 3,61$ $\mathrm{AFE}_{\mathrm{B}}=290,7317-14,4021 \mathrm{DT}+0,2043 \mathrm{DT}^{2} \quad 0,54 * * * 4,39$ $\mathrm{AFE}_{\mathrm{T}}=266,1581-12,8950 \mathrm{DT}+0,1810 \mathrm{DT}^{2} \quad 0,66 * * * 3,24$

AFP: área foliar proyectada $\left(\mathrm{m}^{2}\right.$ árbol $\left.{ }^{-1}\right)$, IAF: índice de área foliar $\left(\mathrm{m}^{2} \mathrm{~m}^{-2}\right)$, AFE: área foliar específica $\left(\mathrm{cm}^{2} \mathrm{~g}^{-1}\right)$. Para cada una de estas variables los subíndices $\mathrm{A}, \mathrm{B}, \mathrm{C}$ y $\mathrm{T}$ corresponden a ápice, centro, base y total de copa, respectivamente. DT: diámetro de tocón $(\mathrm{cm})$; DBCV: diámetro en la base de la copa viva $(\mathrm{cm}) ; \ln$ : logaritmo; $\mathrm{R}^{2}$ : coeficiente de determinación $(*=P<0,05 ; * *=P<0,01 ; * * *=P<0,001$; $\mathrm{ns}=$ no significativo); EEE: error estándar de estimación. Todos los coeficientes de las funciones son significativos $(P<0,05)$, excepto los indicados con ${ }^{\dagger}$.

\section{DISCUSIÓN}

Área foliar proyectada. Los menores valores de AFP se registran en los tratamientos de más alta densidad (1.100 árboles ha-1) (cuadro 1). Esto se explica porque los árboles presentan copas más reducidas y, por lo tanto, con menor área foliar. La distribución del AFP entre las secciones de la copa del árbol es, en todos los tratamientos, proporcionalmente mayor en la sección central (cuadro 1). Esto también ha sido reportado por Medhurst y Beadle (2001) en rodales raleados y no raleados de E. nitens; por Rodríguez (2002) en rodales raleados de $P$. radiata y por Gajardo (2005) en rodales con dos raleos de Nothofagus dombeyi (Mirb.) Oerst.

La intensidad de raleo afectó significativamente el AFP total y por sección de copa (cuadro 2). Este efecto también ha sido registrado en otras especies: en Pseudotsuga menziesii (Mirb.) Franco por Barclay et al. (1986), en $N$. dombeyi por Gajardo (2005) y en P. radiata por Rodríguez (2002). Ello pudiera ser atribuible a una mayor luminosidad disponible para el árbol, provocando un aumento de follaje especialmente en la base de la copa, que permite que las ramas basales continúen creciendo (Medhurst y Beadle 2001). Al contrario de lo observado en el raleo, la poda no tuvo efecto sobre el IAF total $(P=0,3590)$ (cuadro 2$)$, lo cual se explicaría por la tardía intervención de poda, que se practicó cuando la plantación tenía seis años de edad $\mathrm{y}$, por lo tanto, de efecto transitorio sobre el área foliar. A pesar de esto, la interacción raleo-poda es significativa para las variables AFP total $(P=0,0226)$ y AFP de la sección de la base de la copa $(P=0,0279)$ (cuadro 2). Esto indica que si bien la poda individualmente no tiene efecto, sí lo tiene de manera combinada con el raleo, afectando el AFP de la base de la copa. La eliminación de ramas basales deficitarias en aporte de fotosintatos, combinada con raleos que aumentan la luminosidad al interior de la plantación, hace posible el incremento de la superficie foliar, especialmente en la base de la copa viva.

Índice de área foliar. Los tratamientos de poda a 3,5 m y raleo a 800 árboles ha ${ }^{-1}(\mathrm{~T} 4)$ y tratamiento sin poda y 1.100 árboles ha ${ }^{-1}(\mathrm{~T} 0)$ presentan IAF significativamente diferente. Todos los otros tratamientos no presentan diferencias significativas entre ellos (cuadro 3 ). Los valores de IAF obtenidos (cuadro 3) se encuentran dentro de los rangos señalados por otros autores. Medhurst y Beadle (2001) determinaron en rodales de E. nitens raleados a densidad residual de 600 árboles ha-1, valores de IAF de $2,23 \mathrm{~m}^{2}$ $\mathrm{m}^{-2}$ y $2,78 \mathrm{~m}^{2} \mathrm{~m}^{-2}$ luego de 6 y 18 meses del raleo (IAF obtenido utilizando equipo Licor LAI 2000). Amponsah et al. (2005) en Pinus contorta Dougl. encontraron IAF de $2,29 \mathrm{~m}^{2} \mathrm{~m}^{-2}$, el cual aumenta a $2,72 \mathrm{~m}^{2} \mathrm{~m}^{-2}$ con fertilizaciones anuales. En sitios de buena calidad $\left(30 \mathrm{~m}^{3} \mathrm{ha}^{-1} \mathrm{ano}^{-1}\right)$, Beadle et al. (1995) señalan que el IAF para Eucalyptus $s p$. está entre 4,5 y $6 \mathrm{~m}^{2} \mathrm{~m}^{-2}$, en cambio, en sitios pobres es menor a $2 \mathrm{~m}^{2} \mathrm{~m}^{-2}$ (Cherry et al. 1998).

El raleo afectó el IAF total $(P=0,0046)$ (cuadro 4). $\mathrm{Al}$ comparar los tratamientos raleados $(800$ y 400 árboles $\mathrm{ha}^{-1}$ ) con los no raleados (1.100 árboles $\left.\mathrm{ha}^{-1}\right)$ se aprecia que los raleados presentan mayor IAF. Similar tendencia como respuesta al raleo ha sido observada en Pinus taeda L. (Guillespie et al. 1994) y en Pseudotsuga menziesii (Barclay et al. 1986), la que pudiera ser atribuible a una mayor luminosidad disponible por árbol, que provoca un incremento del tamaño de la copa, como también la modificación del estatus hídrico y nutricional del suelo. Al respecto, Barclay et al. (1986) señalan que el desarrollo de follaje depende principalmente de la penetración de luz y de la disponibilidad de nutrientes y agua, lo cual explicaría la mayor superficie foliar de los árboles presentes en tratamientos raleados.

La poda no tuvo efecto sobre el IAF total $(P=0,622)$. Se reconoce que la intervención de poda, especialmente cuando se cortan ramas verdes, altera el área foliar durante un periodo limitado de tiempo. En efecto, el IAF de una plantación de E. nitens se redujo desde 6,3 a $3,5 \mathrm{~m}^{2} \mathrm{~m}^{-2}$ al podar el 50\% de la copa viva, aunque aumentó a 5,5 $\mathrm{m}^{2} \mathrm{~m}^{-2}$ luego de 13 meses de practicada la poda (Pinkard et al. 1999). Eucalyptus nitens, al igual que muchas otras especies, compensa la pérdida de follaje cambiando su patrón de localización de los recursos en favor del desarrollo 
de área foliar. La magnitud y duración de los cambios se incrementa con la severidad de poda (Pinkard y Beadle 1998). En este estudio, la ausencia de efecto de la poda sobre el IAF se explicaría por lo transitorio del efecto de la poda y por lo tardío de la intervención. Mohammed et al. (2000) sugieren que la poda de ramas verdes en $E$. nitens se debe realizar tempranamente, antes del cierre de copas (2,5 años de edad), especialmente en sitios de alta productividad, como es el caso del ensayo establecido en Los Álamos.

La distribución del IAF entre las secciones de la copa del árbol es, en todos los tratamientos, proporcionalmente mayor en la sección central, presentando diferencia significativa sólo entre los tratamientos T0 y T5 (cuadro 3). La localización de una mayor proporción de follaje en la parte central de la copa ha sido reportada por Medhurst y Beadle (2001) en E. nitens, por Rodríguez (2002) en $P$. radiata y por Gajardo (2005) en $N$. dombeyi.

En plantaciones de Eucalyptus spp. el IAF se incrementa con el desarrollo del rodal hasta que se estabiliza o declina levemente (Battaglia et al. 1998). En este estudio el IAF se incrementa desde la densidad 400 a 800 árboles $\mathrm{ha}^{-1}$, para luego decrecer. El análisis teórico señala que el IAF se estabiliza a cierto nivel de luminosidad y tasa de respiración del follaje, nivel que no se ha alcanzado en los árboles de densidad 400 árboles ha ${ }^{-1}$ debido a que aún no logran la cobertura total del dosel.

Por otra parte, árboles en fuerte competencia por luz presentan una mayor asignación proporcional de fotosintatos en el fuste (Nilsson y Albrektson 1993, Naidu et al. 1998), generando con ello una mayor eficiencia de crecimiento (Muñoz 2006). Esto significa que, en algún momento de su crecimiento, los árboles cambian su patrón de asignación de fotosintatos desde la copa al fuste. En el presente estudio el mayor valor de IAF se alcanza a la densidad 800 árboles ha ${ }^{-1}$, para luego decrecer a medida que aumenta la densidad residual. El índice de eficiencia de crecimiento, definido como el incremento de biomasa fustal por unidad de área foliar (Waring et al. 1980), es mayor para la densidad 1.100 árboles ha-1 (Muñoz 2006).

Área foliar específica. El AFE total no presenta diferencias significativas entre tratamientos (cuadro 3), resultado que también se ha registrado en otros estudios (Beets y Lane 1987, Gajardo 2005). Los valores de AFE obtenidos en este estudio se encuentran dentro de los rangos señalados por otros autores. En E. nitens, en plantas de pocas semanas, el AFE varía aproximadamente entre 40 y $50 \mathrm{~cm}^{2} \mathrm{~g}^{-1}$ (Close y Beadle 2003) y a los 7 años, entre 47,5 y $49,3 \mathrm{~cm}^{2} \mathrm{~g}^{-1}$ para densidades de 500 y 1.667 árboles ha-1, respectivamente (Pinkard y Neilsen 2003). Esto indicaría que en $E$. nitens el AFE no cambia de manera significativa al variar la edad y densidad de rodal. En este estudio el AFE es mayor en los tratamientos de densidad 1.100 árboles ha ${ }^{-1}$ (cuadro 4), aunque las diferencias no son significativas. Como señalan Meadows y Hodges (2002), árboles bajo condiciones de menor luminosidad (mayor densidad) tienen mayor área foliar por unidad de peso seco de hojas o una mayor proporción de hojas de sombra. La mayor AFE encontrada en los tratamientos de mayor densidad (1.100 árboles $h^{-1}$ ) se explica por la estrategia que desarrollan los árboles para captar mayor cantidad de luz a través de hojas comparativamente más grandes.

En la mayoría de los tratamientos los valores más altos de AFE se localizan en el tercio basal de la copa, aunque las diferencias son mínimas (cuadro 4). Esto se explica porque el raleo aumenta la capacidad fotosintética de la copa, principalmente en su base, debido a la mejora en las condiciones de luz (Medhurst y Beadle 2000), lo cual está influenciado por el régimen silvicultural (Rodríguez 2002). Sólo hay diferencia significativa entre los tercios apicales de los árboles al comparar el nivel de densidad 400 con 1.100 árboles ha-1 (cuadro 4). Esto es concordante con lo señalado anteriormente, en cuanto a que árboles de copa reducida generan hojas más grandes que les permitan captar más luz.

El incremento del AFE a niveles mayores de densidad ha sido también registrado en Picea abies (Eckmüllner y Sterba 2000), en Pseudotsuga menziesii, Pinus ponderosa Dougl. ex Laws y Pinus monticola Dougl. ex D. Don (Borghetti et al. 1986, Bartelink 1996, Monserud y Marshall 1999) y en Quercus falcata Michx. y Fraxinus pennsylvanica Marsh. (Meadows y Hodges 2002). En estas especies, al igual que en E. nitens en este estudio, la tendencia es que el follaje más sombreado tenga mayor AFE, lo que se explicaría por la necesidad de incrementar la superficie capaz de capturar la luminosidad difusa de sectores sombríos, involucrando a su vez un costo mínimo de respiración (Gajardo 2005).

Funciones alométricas. La variable que mejor estima el AFP es el diámetro en la base de la copa viva, en su expresión logarítmica, con coeficientes de determinación de 0,50 y $0,82(P<0,01)$ para el ápice y total, respectivamente (cuadro 5). Las variables que mejor estiman el IAF son el diámetro de tocón y el diámetro en la base de la copa viva. La mejor correlación del IAF con variables que expresan el tamaño del árbol, fue también registrada por Cherry et al. (1998), y Pinkard y Nielsen (2003) en E. nitens, y Rodríguez (2002) en $P$. radiata. En diversos estudios se ha determinado que la mayor capacidad predictora del área foliar está dada por las variables área de albura en el DAP o en la base de la copa viva, siendo superior una $\mathrm{u}$ otra, de acuerdo con la especie, sitio, tasa de crecimiento y densidad (Kaufman y Troendle 1981, Espinosa y Perry 1987, Gajardo 2005). En este estudio la variable diámetro en la base de la copa viva presentó la mejor correlación entre varias variables probadas (DAP, diámetro del tocón, largo de la copa viva, altura total, además de sus transformaciones cuadráticas y logarítmicas).

Los niveles de correlación obtenidos se explican por la alta variabilidad de los datos. El análisis teórico señala que 
el IAF se estabiliza a un determinado nivel de luminosidad y tasa de respiración del follaje, lo que corresponde al cierre del dosel, situación que aun no se observa en los tratamientos de densidad 400 árboles ha $^{-1}$. El ajuste, con el mismo modelo, de los datos $(n=18)$ correspondientes sólo a los tratamientos con densidades de 1.100 y 800 árboles $\mathrm{ha}^{-1}$, incrementó el $\mathrm{R}^{2}$ de 0,46 a 0,80 (74\%) y disminuyó el error (EEE) de 0,74 a 0,51 (31\%).

La información recolectada en el estudio permite su uso en modelos de procesos ecofisiológicos, como el modelo 3PG (Landsberg y Waring 1997), y dan una explicación más funcional del comportamiento horizontal y vertical del área foliar en rodales con diferentes densidades residuales.

\section{CONCLUSIONES}

El raleo tuvo efecto significativo en el área foliar proyectada e índice de área foliar. El área foliar proyectada e índice de área foliar es máxima en los tratamientos de densidad 400 árboles ha $^{-1}$ y en tratamientos de densidad 800 árboles ha-1, respectivamente. Luego de alcanzar el máximo, el índice de área foliar disminuye debido a la menor proporción de biomasa localizada en la copa de los árboles. El área foliar específica es mayor en tratamientos de densidad de 1.100 árboles $\mathrm{ha}^{-1}$, sin efecto significativo entre tratamientos. El área foliar proyectada e índice de área foliar se concentran en la sección central de la copa. Los modelos alométricos determinados utilizan como variable predictora el diámetro en la base de la copa viva y el diámetro de tocón.

\section{AGRADECIMIENTOS}

Se agradece el apoyo de la empresa Bosques Arauco S.A. para desarrollar la investigación, al editor y revisores anónimos por las observaciones realizadas al manuscrito.

\section{REFERENCIAS}

Amponsah I, P Comeau, R Brockley, V Lieffers. 2005. Effects of repeated fertilization on needle longevity, foliar nutrition, effective leaf area index, and growth characteristics of lodgepole pine in interior British Columbia, Canada. Can. J. For. Res. 35: 440-451.

Barclay HJ, PC Pang, FW Pollard. 1986. Aboveground biomass distribution within trees and stand in thinned and fertilized Douglas-fir. Can. J. For. Res. 16: 438-442.

Bartelink H. 1996. A model of dry matter partitioning in trees. Tree Physiology 18: 91-101.

Battaglia M, ML Cherry, CL Beadle, PJ Sands, A Hingston. 1998. Prediction of leaf area index in eucalypt plantations: effects of water stress and temperature. Tree Physiology 18: 521-528.
Beadle CL, JL Honeysett, C Turnbull, D White. 1995. Site limits to achieving genetic potential. In Potts $\mathrm{B}, \mathrm{N}$ Borralho, J Reid, R Cromer, W Tibbits, C Raymond eds. Eucalypt Plantations: Improving Fibre Yield and Quality. Proceedings CRC Temperate Hardwood Forestry - IUFRO Conference, 19-24 Feb 1995. Hobart, Australia. p. 325-331.

Beets P, P Lane. 1987. Specific leaf area of Pinus radiata as influenced by stand age, leaf age, and thinning. N. Z. J. For. Sci. 17(2/3): 283-291.

Borghetti M, GG Vendramin, R Giannini. 1986. Specific leaf area and leaf area index distribution in a young Douglas-fir plantation. Can. J. For. Res. 16: 1283-1288.

Carrasco P, J Millán. 1990. Proyecto de suelos forestales de la VIII Región. Informe final. Chillán, Chile. Universidad de Concepción, Depto. de Cs. Forestales/Min. de Agricultura. $152 \mathrm{p}$.

Cherry M, A Hingston, M Battaglia, C Beadle. 1998. Calibrating the LI-COR LAI-2000 for estimating leaf area index in eucalypt plantations. Tasforests 10: 75-82.

Cherry M, C Macfarlane, P Smethurst, C Beadle. 2002. Visual guide to leaf area index of Eucalypt plantations. Hobart, Australia. Cooperative Research Centre for Sustainable Production Forestry. $24 \mathrm{p}$.

Close D, C Beadle. 2003. Chilling-dependent photoinhibition, nutrition and growth analysis of Eucalyptus nitens seedlings during establishment. Tree Physiology 23: 217-226.

Eckmüllner O, H Sterba. 2000. Crown condition, needle mass, and sapwood area relationship of Norway spruce (Picea abies). Can. J. For. Res. 30: 1646-1654.

Espinosa M, D Perry. 1987. Distribution and increment of biomass in adjacent young Douglas-fir stands with different early growth rates. Can. J. For. Res. 17: 722-730.

Gajardo P. 2005. Efectos del raleo y clase de copa sobre el área foliar, la distribución del crecimiento y la eficiencia del crecimiento en árboles individuales de coigüe. Tesis de Magíster en Ciencias Forestales. Concepción, Chile. Universidad de Concepción. 60 p.

Guillespie A, HL Allen, J Vose. 1994. Amount and vertical distribution of foliage of young loblolly pine trees as affected by canopy position and silvicultural treatment. Can. J. For. Res. 24. 1337-1344.

INFOR (Instituto Forestal, CL). 2004. Eucalyptus nitens en Chile: Primera monografía. Informe Técnico $N^{\circ} 165$. Valdivia, Chile. Instituto Forestal. 143 p.

Jerez M, T Dean, Q Cao, S Roberts. 2005. Describing leaf area distribution in Loblolly Pine trees with Johnson's SB function. Forest Science 51(2): 93-101.

Kaufman MR, CA Troendle. 1981. The relationship of leaf area and foliage biomass to sapwood conducting area in four subalpine forest tree species. Forest Science 27: 477-486.

Keane R, E Reinhardt, J Scott, K Gray, J Reardon. 2005. Estimating forest canopy bulk density using six indirect methods. Can. J. For. Res. 35: 724-739.

Landsberg, JJ, RH Waring. 1997. A generalised model of forest productivity using concepts of radiation use efficiency, carbon balance and partitioning. Forest Ecology and Management 95: 209-228.

Meadows J, J Hodges. 2002. Sapwood area as an estimator of leaf area and foliar weight in Cherrybark Oak and Green Ash. Forest Science 48(1): 69-76. 
Medhurst J, C Beadle. 2000. Thinning for solid wood products in Eucalyptus nitens plantations. In Henderson L, G Waugh, G Nolan, P Bennett eds. The Future of Eucalypts for Wood Products. Proceedings of IUFRO Conference, 19-24 March 2000. Launceston, Australia. p. 343-348.

Medhurst J, C Beadle. 2001. Crown structure and leaf area index development in thinned and unthinned Eucalyptus nitens plantations. Tree Physiology 21: 989-999.

Mohammed C, K Barry, M Battaglia, C Beadle, A Eyles, A Mollon, E Pinkard. 2000. Pruning-associated stem defects in plantation E. nitens and E. globulus grown for sawlog and veneer in Tasmania, Australia. In Henderson L, G Waugh, G Nolan, P Bennett eds. The Future of Eucalypts for wood products. Proceedings IUFRO Conference, 19-24 March 2000. Launceston, Australia. p. 357-364.

Monserud R, J Marshall. 1999. Allometric crown relations in three northern Idaho conifer species. Can. J. For. Res. 29: 521-535.

Muñoz F, M Espinosa, MA Herrera y J Cancino. 2005. Características del crecimiento en diámetro, altura y volumen de una plantación de Eucalyptus nitens sometida a tratamientos silvícolas de poda y raleo. Bosque 26(1): 93-99.

Muñoz F. 2006. Eficiencia de crecimiento y tratamientos silviculturales en E. nitens. In Sociedad Chilena de Ciencias Forestales - Universidad de Concepción eds. I Simposio de Silvicultura Intensiva de Plantaciones, 28-30 Nov 2006. Concepción, Chile. p. 236.

Naidu SL, E DeLucia, R Thomas. 1998. Contrasting patterns of biomass allocation in dominant and suppressed loblolly pine. Can. J. For. Res. 28: 1116-1124.

Nilsson U, A Albrektson. 1993. Productivity of needles and allocation of growth in young Scots pine trees of different competitive status. Forest Ecology and Management 62: 173-187.

Norman JM, GS Campbell. 1989. Canopy structure. In Pearcy RW, J Ehleringer, HA Mooney, PW Rundel eds. Plant physiological ecology: Field methods and instrumentation. London, UK. Chapman and Hall. p. 301-325.

Oliver CD, BC Larson. 1996. Forest stand dynamics (Update edition). New York, USA. John Wiley and Sons. 520 p.

Pardé DR. 1980. Forest biomass. Review article. Forest Products Abstract 41: 343-362.
Perry D. 1994. Forest ecosystem. Baltimore, USA. The Johns Hopkins University Press. 649 p.

Pinkard E, M Battaglia, C Beadle, P Sands. 1999. Modelling the effect of physiological responses to green pruning on net biomass production of Eucalyptus nitens. Tree Physiology 19: $1-12$.

Pinkard E, C Beadle. 1998. Regulation of photosynthesis in Eucalyptus nitens (Deane and Maiden) Maiden following green pruning. Trees 12: 366-376.

Pinkard E, C Beadle, N Davidson, M Battaglia. 1995. Changes in crown productivity and stem growth of Eucalyptus nitens in response to green pruning. In Potts $\mathrm{B}, \mathrm{N}$ Borralho, $\mathrm{J}$ Reid, R Cromer, W Tibbits, C Raymond eds. Eucalypt Plantations: Improving Fibre Yield and Quality. Proceedings CRC Temperate Hardwood Forestry - IUFRO Conference, 19-24 Feb 1995. Hobart, Australia. p. 377-378.

Pinkard E, E Neilsen. 2003. Crown and stand characteristics of Eucalyptus nitens in response to initial spacing: implications for thinning. Forest Ecology and Management 172: 215-227.

Rodríguez R. 2002. Effects of silvicultural regime on leaf, allometry, growth allocation and productivity in Pinus radiata D. Don. Tesis de Doctorado en Ciencias Forestales. Concepción, Chile. Universidad de Concepción. 109 p.

Santibáñez F, J Uribe. 1993. Atlas agroclimático de Chile, regiones Sexta, Séptima, Octava y Novena. Santiago, Chile. Corporación de Fomento, Ministerio de Agricultura, FIA. 99 p.

Simioni G, J Gignoux, X Le Roux, R Appé, D Benest. 2004. Spatial and temporal variations in leaf area index, specific leaf area and leaf nitrogen of two co-occurring savanna tree species. Tree Physiology 24: 205-216

Smethurst P, C Baillie, M Cherry, G Holz. 2003. Fertilizer effects on LAI and growth of four Eucalyptus nitens plantations. Forest Ecology and Management 176: 531-542.

STATSOFT INC. 2001. STATISTICA (data analysis software system), version 6. www.statsoft.com.

Steel R, J Torrie. 1988. Bioestadística: Principios y procedimientos. México DF. México. McGraw-Hill/Interamericana. 622 p.

Waring RH, WG Thies, D Muscato. 1980. Stem growth per unit of leaf area: a measure of tree vigor. Forest Sci. 26: 112-117. 